\title{
Protée
}

\section{La science de Guy Blackburn}

\section{Christine Martel}

Volume 37, numéro 3, hiver 2009

Regards croisés sur les images scientifiques

URI : https://id.erudit.org/iderudit/038807ar

DOI : https://doi.org/10.7202/038807ar

Aller au sommaire du numéro

Éditeur(s)

Département des arts et lettres - Université du Québec à Chicoutimi

ISSN

0300-3523 (imprimé)

1708-2307 (numérique)

Découvrir la revue

Citer cet article

Martel, C. (2009). La science de Guy Blackburn. Protée, 37(3), 75-80.

https://doi.org/10.7202/038807ar d'utilisation que vous pouvez consulter en ligne.

https://apropos.erudit.org/fr/usagers/politique-dutilisation/ 


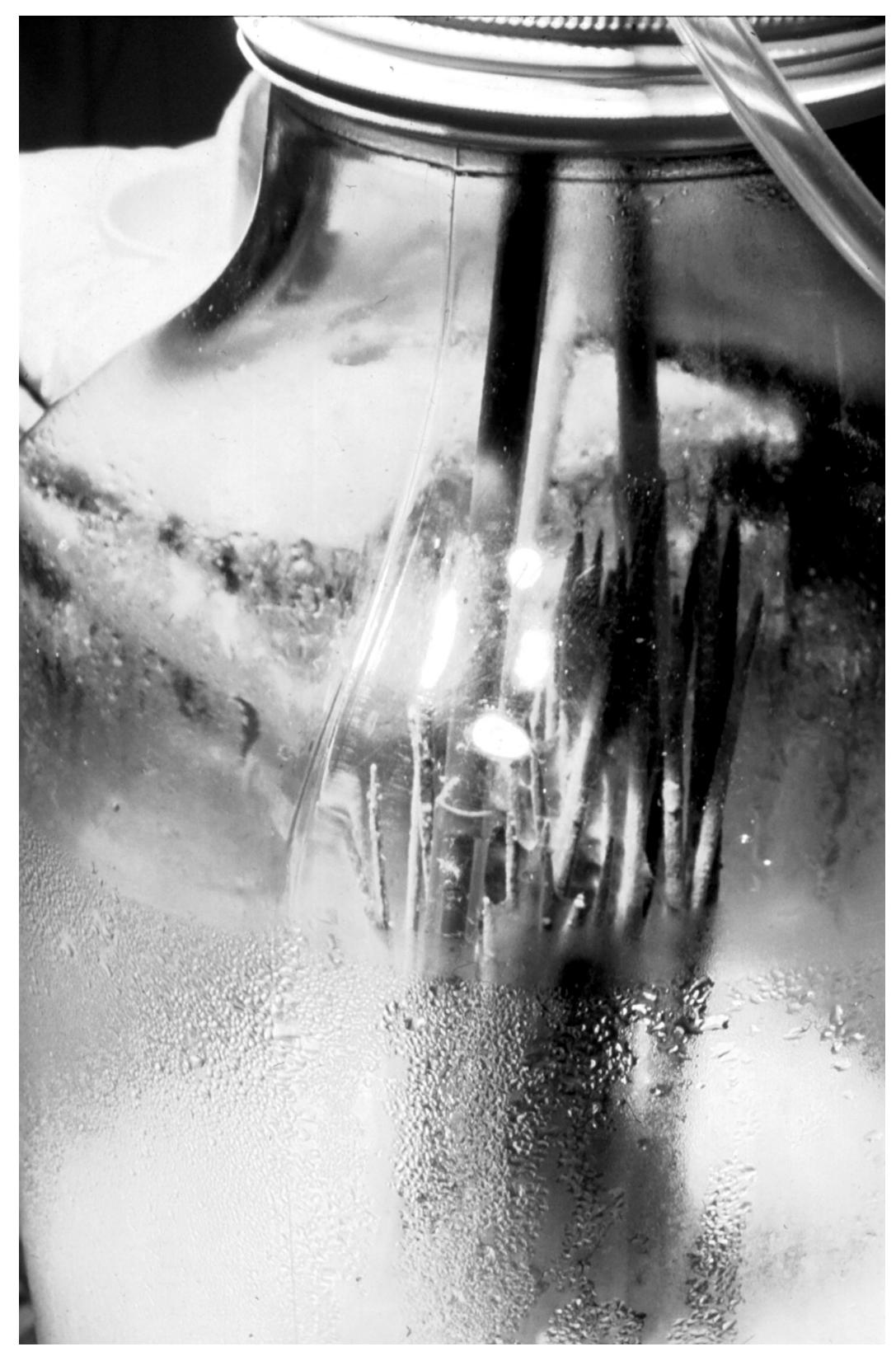




\section{LA SCIENCE DE GUY BLACKBURN}

$\mathrm{S}$

i l'on se permet aisément d'affirmer que l'art est politique, sociologique ou même thérapeutique, c'est de toute évidence plus risqué de le comparer à la science. Il est pourtant logique et vraisemblable de prétendre que ces deux domaines entretiennent des rapports comme constituants d'une même culture. Mais cette association probable semble en général très délicate et entraîne plus souvent qu'autrement la négation du rapprochement. Pourtant certains artistes, comme des scientifiques voués à la poursuite d'une objectivation des faits, s'engagent bel et bien dans une recherche conceptuelle et formelle construite d'observation et d'expérimentation. Bien que consacré à l'expression d'un idéal esthétique, et d'un instinct de l'harmonie, leur art se destine en ce sens à exprimer une vision d'un univers à l'intérieur duquel la science se déploie.

L'art rechercherait la beauté; la science, la vérité. L'un parlerait à l'émotion, l'autre à la raison. Mais leurs similitudes sont en fait plus réelles qu'il n'y paraît. En effet, I'un et l'autre expriment la pensée par la technique. Dans les deux cas, l'imagination ainsi maîtrisée permettrait à chacun de se dépasser. En science comme dans les arts, on réclame des correspondances avec une réalité qui reflète la société ou qui la subvertit. S'il est dit des entreprises de Guy Blackburn qu' elles « explorent de façon percutante, voire troublante, les nouvelles conditions individuelles et sociales issues des récentes pratiques scientifiques ${ }^{1}$, il est permis de croire que l'espace que cet artiste « occupe» soit le même que celui des gens de science.

Comme pour les scientifiques, le point de démarrage des études de Blackburn naît de l'identification d'un problème et de la prise de position. Celui qu'on appelle volontiers « éveilleur de consciences", en mettant sous la loupe sa désapprobation d'une certaine éthique du monde, prend le même risque que tout chercheur qui est au service d'une hypothèse de départ: dépendre des facteurs qui peuvent influencer les retombées. À cette fin, pour chaque projet, I'artiste recueille un lot étonnant de renseignements disponibles qui sont associés à son sujet. À partir de toutes ces données, il prédit les résultats de l'examen effectué dans l'objectif de leur donner une direction. En chemin, si certaines affirmations se révèlent fausses à ses yeux, elles seront le tremplin à une série d'autres questions qu'il faudra un jour ou l'autre aborder et résoudre.

Christine Martel

1. Extrait du catalogue Touche de Guy Blackburn, publé par le Centre Sagamie, Alma (2009: 66). 


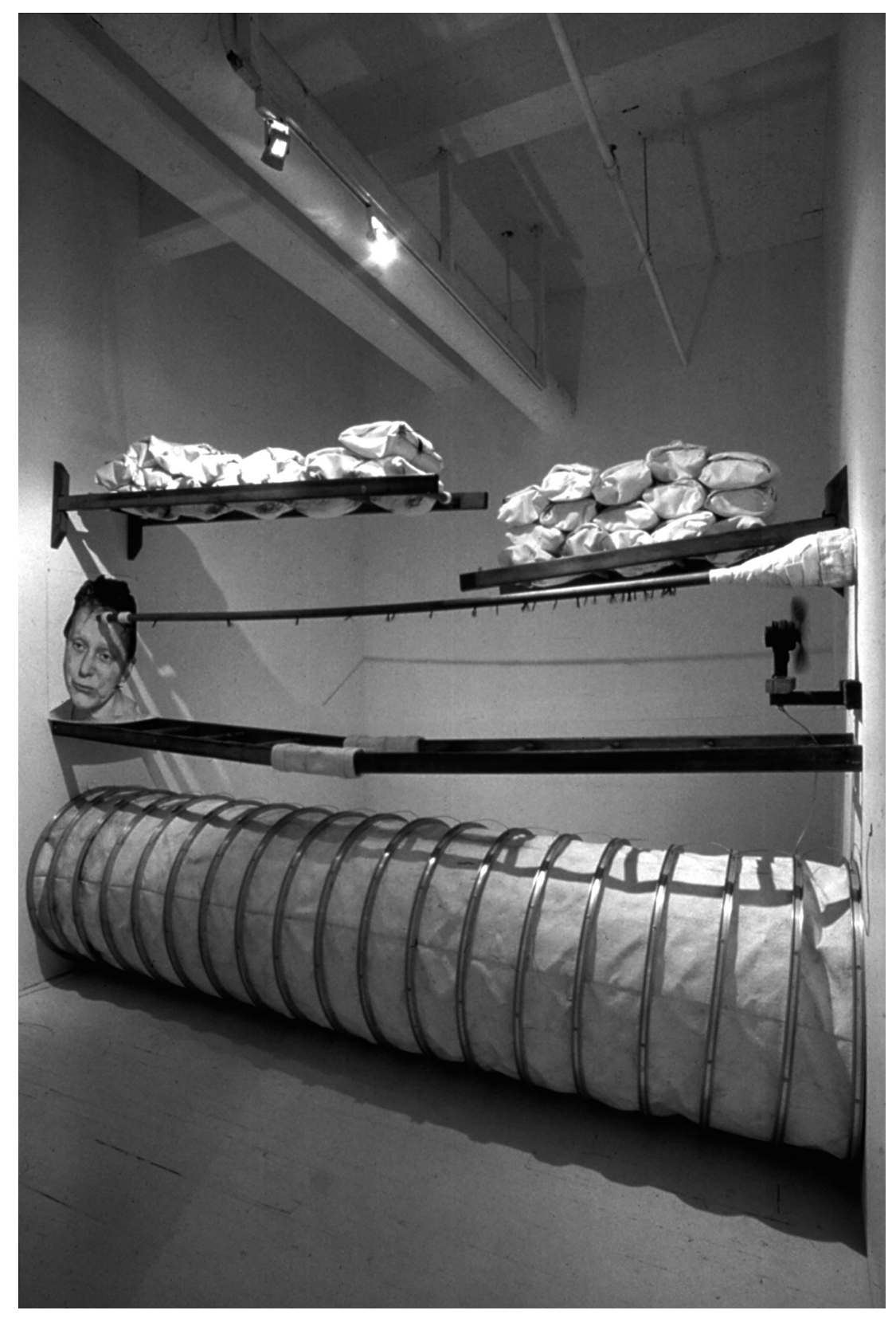



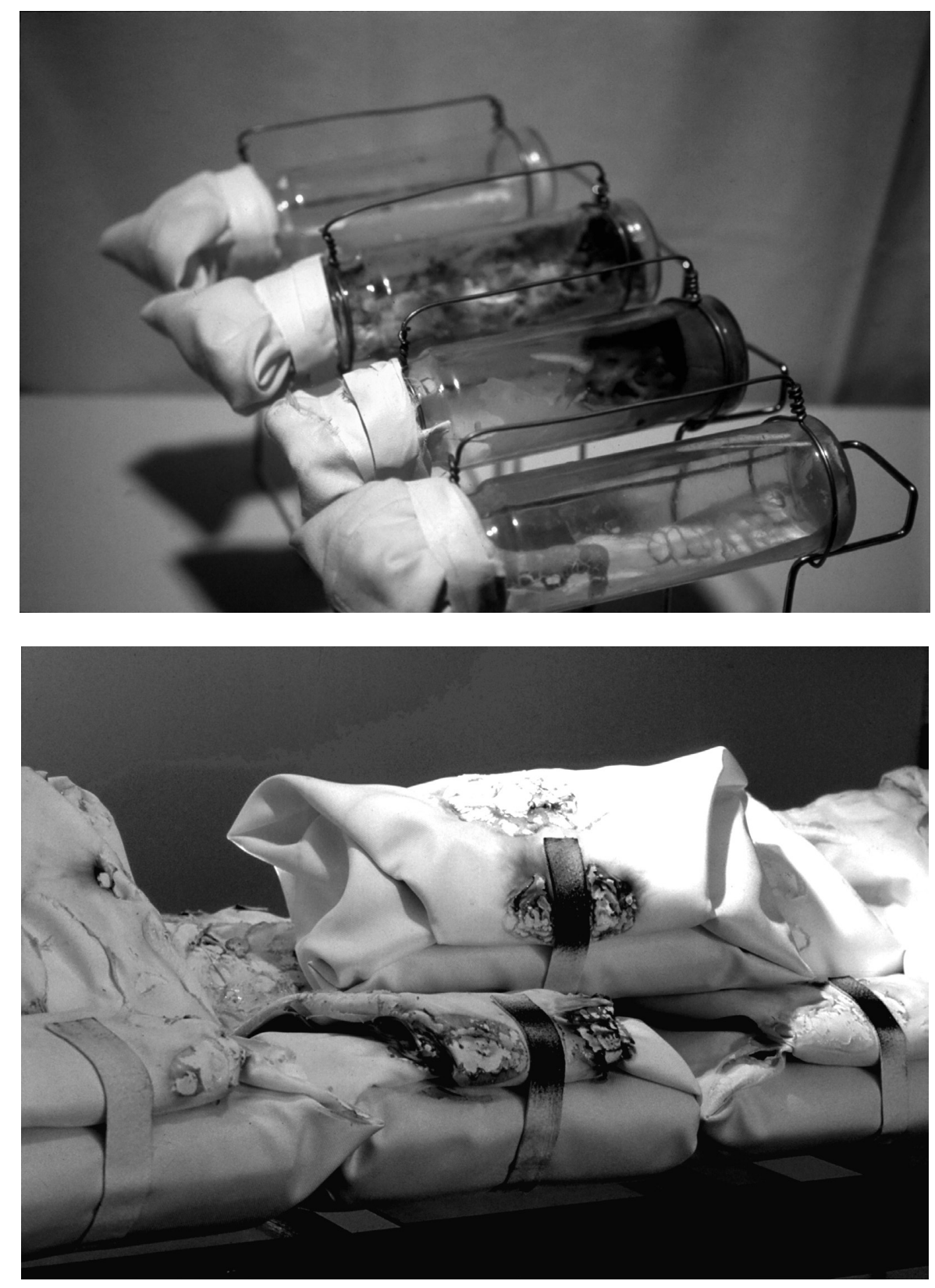


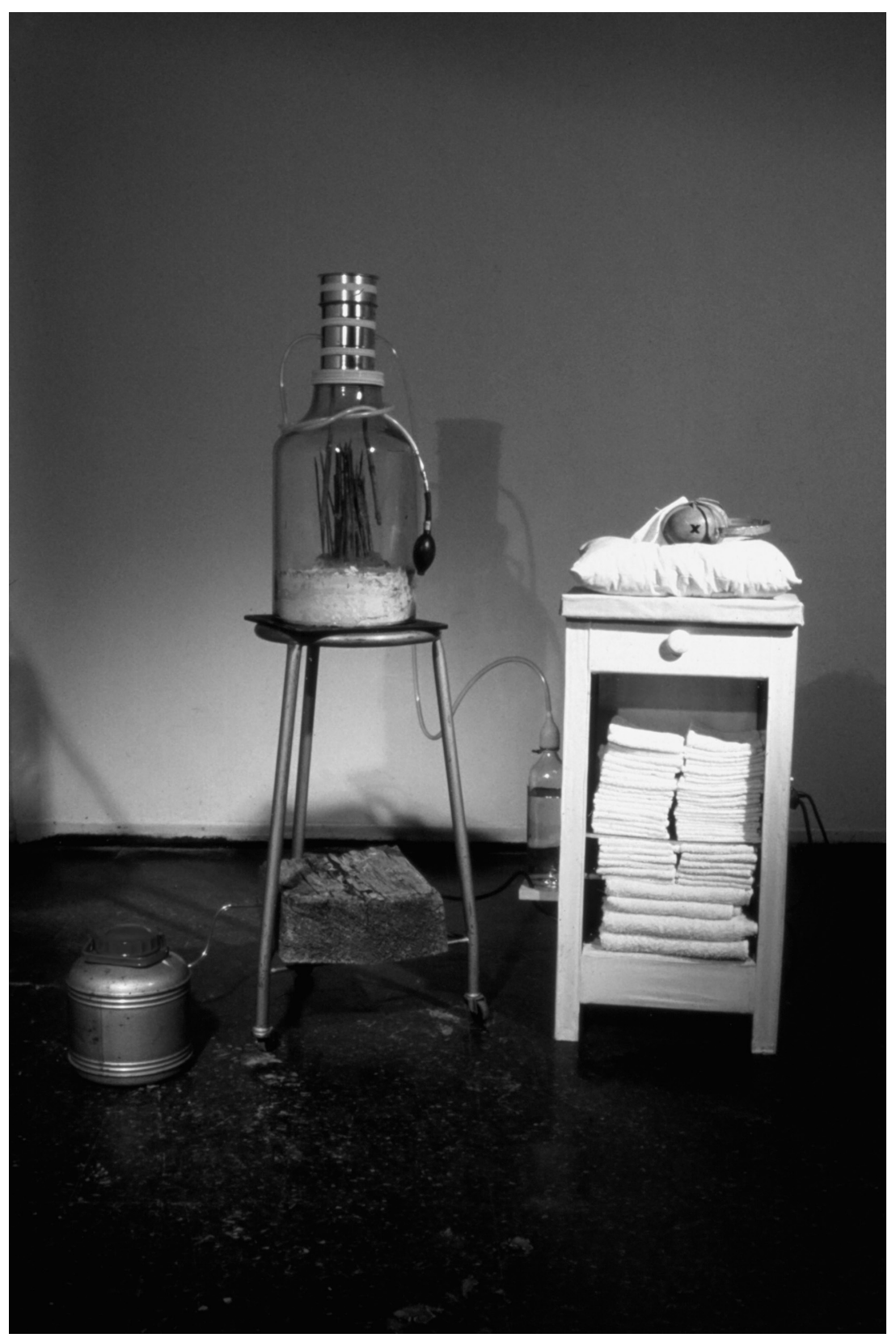




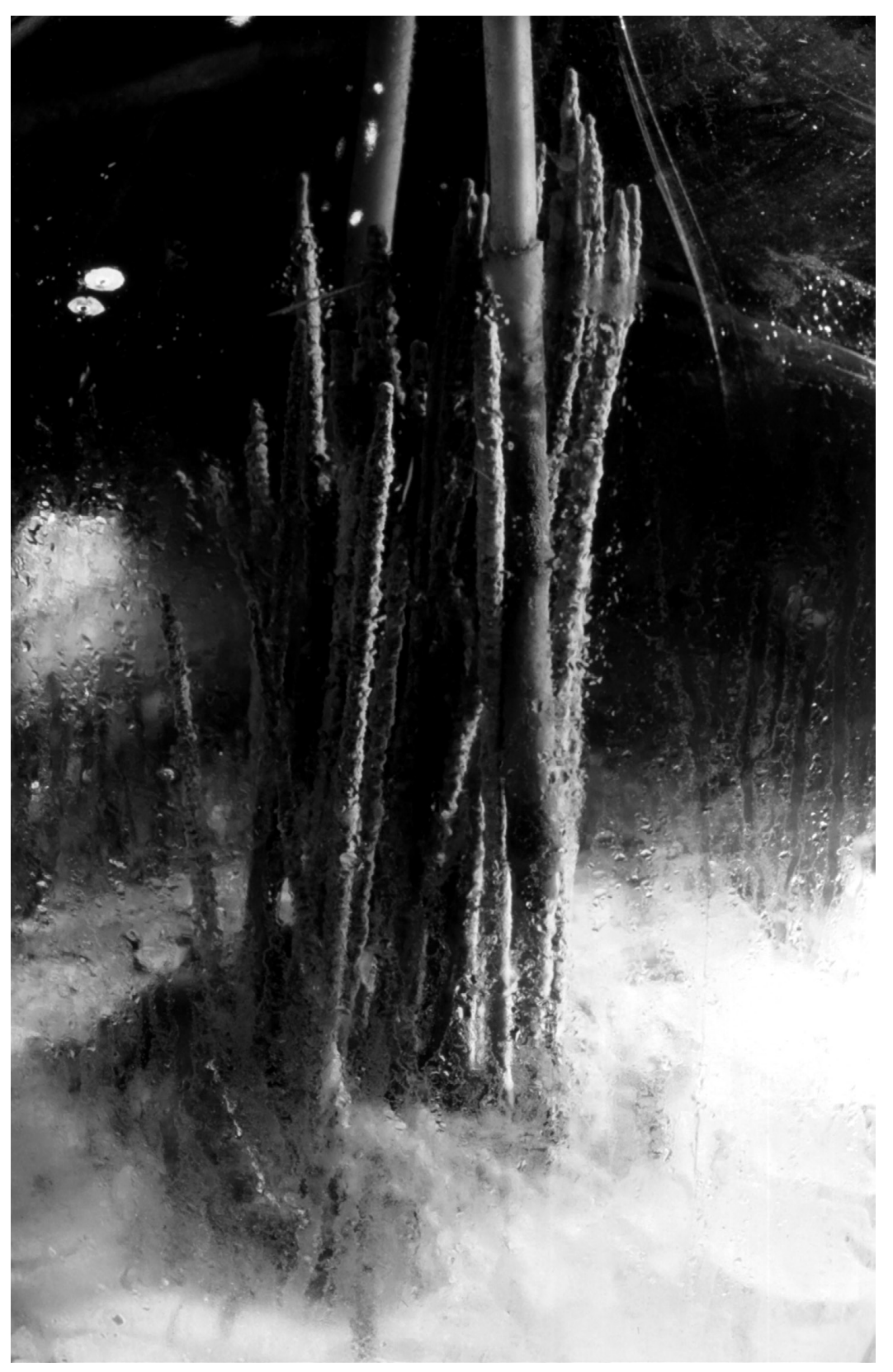


Page 74 «Hommage à Anna (solitude et asepsie)», 1995 ; page 76 « Hommage à Anna (solitude et asepsie)», 1995 ; page 77 «Les grands perturbateurs» (détail), 1992; «Espace inédit (solitude et asepsie)» (détail), 1996; page 78 «Hommage à Anna (solitude et asepsie)» (détail), 1996; page 79 « Hommage à Anna (solitude et asepsie)» (détail), 1995 ; page 80 « Espace inédit (solitude et asepsie)», 1995.

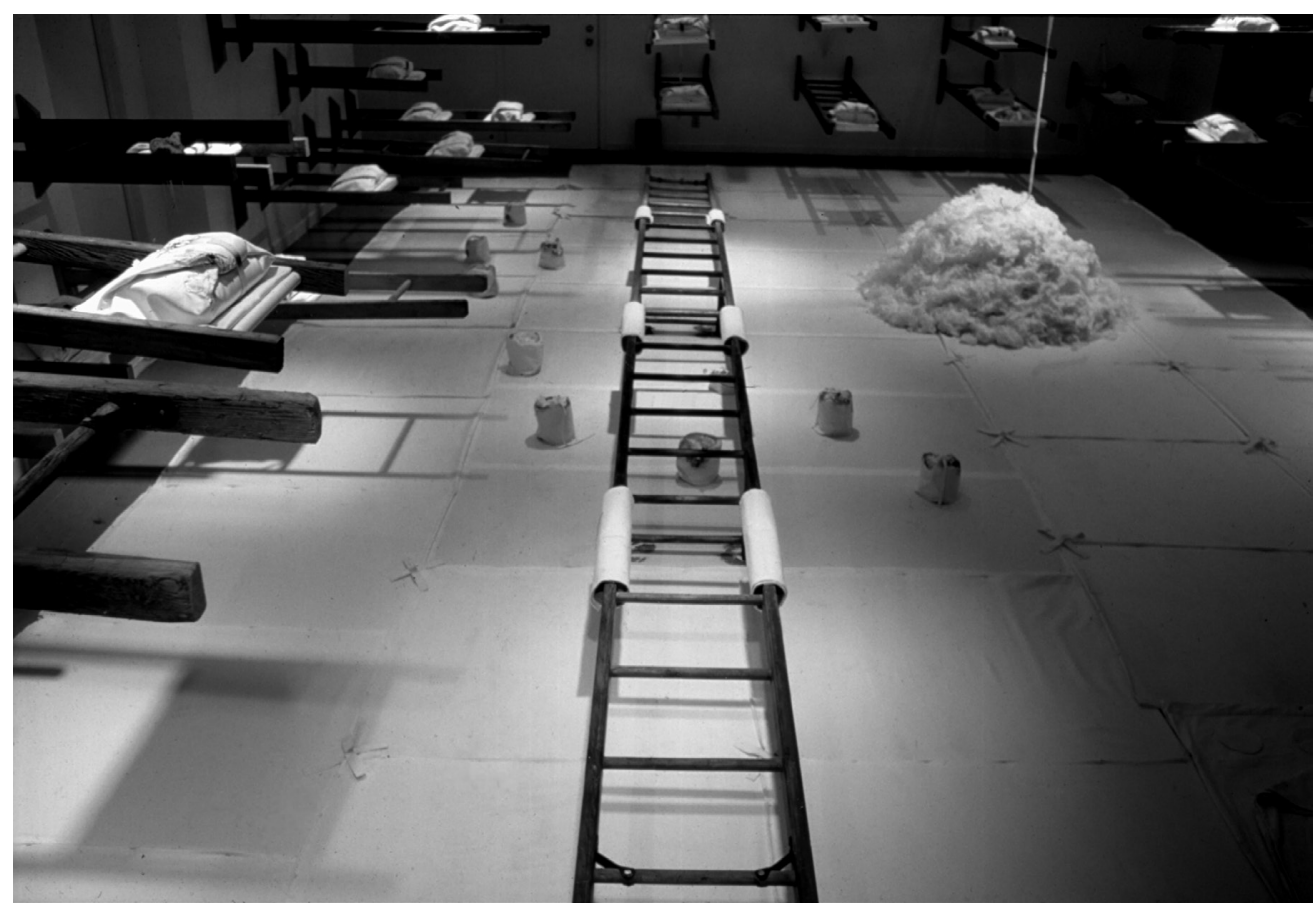

